\section{Assessment of the Anticoagulation Activity of Apixaban}

\section{To the Editor:}

In the February 2015 issue of the Circulation Journal, Silvain et $\mathrm{al}^{1}$ review the available laboratory tests to evaluate the anticoagulant effects of non-vitamin $\mathrm{K}$ antagonist oral anticoagulants (NOACs). To assess the anticoagulant activity of apixaban, they show that prothrombin time (PT/INR) and activated partial thromboplastin time (aPTT) are inadequate and should not be recommended. The test that should be used is a modified anti-Xa using specific calibrators and controls. ${ }^{1}$ These recommendations are according to most of the published data on apixaban laboratory evaluation tests. ${ }^{2}$ However, in the figure published in their article, thrombin-clotting time (TT) is presented as a third choice for apixaban. ${ }^{1}$

In studies performed in order to determine which coagulation assay could be used to assess the effect of apixaban on hemostasis, TT was evaluated by a chronometric method, using reagents from Diagnostica Stago and it was shown that TT is not influenced by apixaban. ${ }^{2}$ Indeed, direct or indirect factor Xa inhibitors have no effect on TT and cannot be recommended for monitoring NOACs anticoagulant activity, as referred by others. ${ }^{3,4}$

NOACs were designed to be given in fixed doses without routine coagulation monitoring. However, in some circumstances, namely when patients need emergency surgery or present with severe bleedings, it is important to have laboratory tests that are able to assess their anticoagulant activity.

Routine coagulation tests, such as aPTT, TT or PT, are nor- mally used to evaluate the anticoagulant activity of dabigatran or rivaroxaban. These tests are easily performed and available $24 \mathrm{~h}$ per day. Because of its expanding use worldwide, it is important to have laboratory tests that are able to assess the anticoagulant activity of apixaban whenever needed, without limitations of time and equipment, as the specific anti-Xa test is not available $24 \mathrm{~h}$ per day in most hospitals.

\section{Grants}

None.

\section{References}

1. Silvain J, Hauguel M, Kerneis M, Collet JP, Montalescot G; for the ACTION Study Group. Measuring and reversing the effect of nonvitamin $\mathrm{K}$ antagonist oral anticoagulants (NOACs) [Editorial]. Circ J 2015; 79: 289-291.

2. Douxfils J, Chatelain C, Chatelain B, Dogné JM, Mullier F. Impact of apixaban on routine and specific coagulation assays: A practical laboratory guide. Thromb Haemost 2013; 110: 283-294.

3. Tobu M, Iqbal O, Hoppensteadt DA, Shultz C, Jeske W, Fareed J. Effects of a synthetic factor Xa inhibitor (JTV-803) on various laboratory tests. Clin Appl Thromb Hemost 2002; 8: 325-336.

4. Baglin T, Keeling D, Kitchen S. Effects on routine coagulation screens and assessment of anticoagulant intensity in patients taking oral dabigatran or rivaroxaban: Guidance from the British Committee for Standards in Haematology. Br J Haematol 2012; 154: $427-$ 429.

Luciana Ricca Gonçalves, MD

Fernando Araújo, MD, PhD

Department of Transfusion Medicine, Centre of Thrombosis and Hemostasis, São João University Hospital, Faculty of Medicine, Porto University, Porto, Portugal

(Released online May 29, 2015) 